\title{
Interactive Automation of COVID-19 Classification through X-Ray Images using Machine Learning
}

\author{
Ashura binti Hasmadi ${ }^{1}$, Mehak Maqbool Memon ${ }^{2}$, Manzoor Ahmed Hashmani ${ }^{3}$
}

\begin{abstract}
Machine learning had given many benefits to the humankind by implementing technology on the daily human lives. To add, when the pandemic COVID19 hits Earth globally in early 2020, mankind is challenged with the sudden emergence of the virus that costed many lives. With the virus spreading fast, it has become a challenge towards medical experts to keep their environment clean from the virus. Scientists and medical experts raced to find a cure and plausible methods to avoid the virus from spreading, ranging from lockdowns to standard operating procedures on daily routines. Studies have also shown that geographical factors in the rural area becomes a great challenge to experts on providing medical attention towards the community that had been infected in the rural areas. Fortunately, with the help of advanced current technology, scientists and medical experts are able to counter these problems. In this study, an experimental model with an accuracy of $87 \%$ is used, and the application to a web server is used via Python and Flask. The accuracy is achieved by adjusting batch sizes and implementing image augmentation using Keras' ImageDataGenerator feature. Therefore, this project focuses on utilizing machine learning to classify COVID-19 patients through X-ray images on a web server, which could further improve the accessibility for humanity to seek for medical attention.
\end{abstract}

Keywords - COVID-19 (Coronavirus Disease), Machine Learning (ML)

Ashura binti Hasmadi

Department of Computer and Information Sciences Universiti Teknologi PETRONAS

Seri Iskandar, Malaysia

(email:ashura.hasmadi_23960@utp.edu.my)

Mehak Maqbool Memon

Department of Computer and Information Sciences

Universiti Teknologi PETRONAS

Seri Iskandar, Malaysia

(email:mehak_19001057@utp.edu.my)

Manzoor Ahmed Hashmani

Department of Computer and Information Sciences

Universiti Teknologi PETRONAS

Seri Iskandar, Malaysia

(email:manzoor.hashmani@utp.edu.my)

\section{INTRODUCTION}

According to the WHO, COVID-19 is a lifethreatening virus that spreads primarily through droplets of saliva or discharge from the nose or mouth, when the infected coughs or sneezes. Other than being a deadly virus itself, COVID-19 also spreads in the human body fast, ranging from asymptomatic to symptomatic infections. Thus, this results to hospitals lacking enough labor to uphold the many patients that needed aid, as well as the increase in front liners getting infected themselves. As the number of infected increases, hospitals are not capable of treating many of those infected, where their hospitals are lacking medical equipment especially those of in the rural areas. In fact, hospitals have become the most dangerous place to visit, and patients are not advised to stay around any longer to avoid getting exposed to the virus. This problem on providing patients a safer environment for testing, makes it a huge challenge for medical team to detect the virus in patients. Scientists have come up with various innovative ways on detecting and identifying COVID19 in human bodies. Generally, COVID-19 can be identified from common symptoms such as fever, cough, fatigue, shortness of breath and loss of smell and taste. For the common medical tools to detect COVID-19, doctors regularly use a device called reverse transcription polymerase chain reaction: RT-PCR. The device detects proteins from the COVID-19 virus in respiratory samples or detection in blood. However, there are issues of where there is a shortage of RT-PCR kits especially in developing countries. Machine learning techniques are used as an alternative to detect certain variables of COVID-19 detection in a much economical way. 


\section{LITERATURE REVIEW}

According to Cleverly et al. [1] and Wong et al. [2], both of their research on chest X-ray images of COVID19 patient has consolidation or ground-glass opacities forming on parts of the chest. Another article from Zhou et al. [3] also stated that the same form of consolidation forms on lung CT images. It is also important to note that most COVID-19 patients do not develop pneumonia therefore it should be distinguishable between a pneumonia infected lung and COVID-19 infected lung.
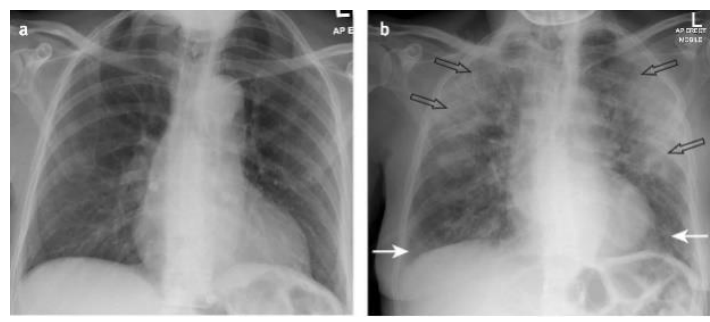

Figure 1. (A) A normal chest radiograph of a patient on her first day of admission in the hospital due to COVID-19. (B) Chest radiograph of the same patient on day 8 showing ground glass opacification on white arrows and consolidation on outlined arrows

Zhou et al. [3], states that in China, CT is the main form of COVID-19 detection in lungs as it has a high reputable sensitivity for the detection. However, another article from Wong et al. [2] suggests that CT scans are a less preferred approach due to how huge and inconvenient for COVID-19 decontamination. Acknowledging that COVID-19 is a highly infectious virus that could survive on non-living objects, medical equipment used must be cleaned each time used. This then would be non-economical and slower down radiology services for COVID-19 detection in lungs. Cleverly et al. [1] also mentioned that portable radiology is the most advisable method to be used in these times to minimize the risk of COVID-19 infection. However, the gaps that could be seen between the two articles from Cleverly et al [1] and Wong et al. [2] is that the observation for each radiology images are done by radiologists. This is rather worrying as there is not many radiologists in this world that could observe every X-ray images of COVID-19 patients, especially in smaller countries.

The challenges faced by radiologists in this pandemic era harms their health as they are required to analyze their patient's result. [4] Asymptomatic infection makes it a greater challenge for doctors and researchers to grant care for their patients, as the identification process gets harder. The issue with COVID-19 is that the virus primarily spreads via droplets via the nose or mouth, and it spreads even rapidly in an area that is not well ventilated. Hospitals have to make sure that they follow up with strict protocols to avoid the infection spread, and this just causes the frontline warriors find difficulty in keeping themselves safe and sanitized, but also challenging to tend to the infected patients. Machines have to be regularly sanitized and face to face meetups needed to be done regularly. This issue serves a huge challenge to CT scanners, despite their high sensitivity in detecting infection in radiology. To accommodate the scarcity of clean expensive CT scanners and keeping the environment safe from the spread of virus, researches have proposed on using mobile $\mathrm{X}$-rays as an alternative choice [5]. From these studies we can identify that by the help of portable devices, the detection of COVID-19 can be executed faster without risking anymore lives for the frontline warriors. Now with the help of portable technology, the medical team have less to worry about time, as the virus infect and could kill the infected at a concerning rate.

The theme for this subtopic is that all of the research and articles agree on using deep learning on the detection of COVID-19 in lungs radiology. Some researches have been made on detecting COVID-19 via deep learning on predicting chest CT images [6], and blood tests [7]. The machine learning application of blood test to the detection of COVID-19 in human body has a distinctive methodology that differs from X-ray and CT scanned image detections. The application of machine learning in blood tests mainly uses logistic regression [8] and random forest classification algorithm [8]. This hold different for image based dataset such as CT scanned images and X-ray images. Deep learning methods such as VGG-19, COVID-Net, ResNet50+ SVM, and DarkCovidNet has been developed by researchers to detect COVID-19 infection for image-based dataset [9]. And as for X-ray detection, many researches have trained and tested their models by using a collected dataset shared by Dr. Joseph Cohen in his GitHub. [10] Researches had been using many different combination of dataset throughout the machine 
learning process in the detection of COVID-19 in lungs $\mathrm{X}$-ray images, since the lack of data could cause bias upon the research [11]. A research by Ozturk, T., et al (2020) had been done with Dr. JP Cohen's open access COVID-19 chest X-ray images, with a dataset containing non-COVID images only belonging to children of 1 to 5 years old. [9] Another research by Sethy, P. et al (2020) that uses Dr. JP Cohen's dataset and a combination of other sources also includes applying the dataset on 11 number of pre-trained CNN models. [12]

\section{METHODOLOGY}

The study has its model built and trained in a Google Colab terminal, and obtained its dataset from Kaggle. [13] After building the model, the model will be saved in an h5 file, and transported to Visual Studio Code where the web application interface will be implemented with Flask.

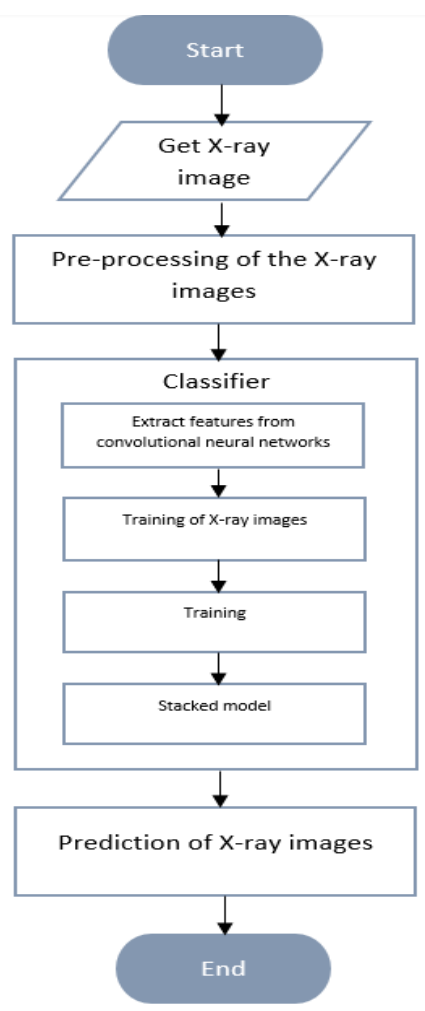

Figure 2. Flowchart of the machine learning process

\section{A. The dataset}

The image database includes image X-rays of Normal, COVID-19 and Viral Pneumonia infected lungs included in the file. As of the now, there are 3616 images of COVID X-rays, 10.2k images of Normal X-rays and 1345 images of Viral Pneumonia X-rays. All images are in the Portable Network Graphic (PNG) file format and has a resolution of 299x299 pixels. Thus, for these research only Normal and COVID-19 X-ray images are used to conduct study. The database is constantly updated and is contributed and filtered by researchers from various regions. The Figure below shows a few examples of the chest X-ray images in the dataset.
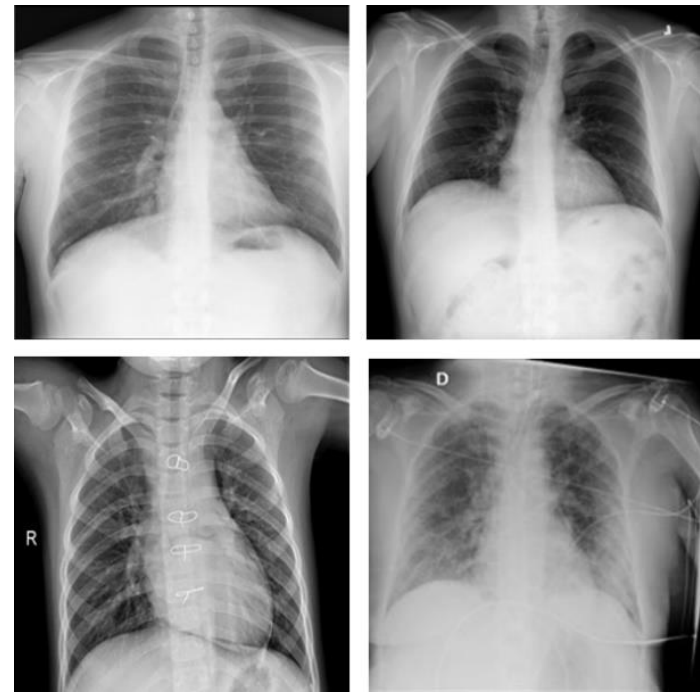

Figure 3. Examples of images in the dataset. Upper Left and Bottom Right both shows COVID X-rays, while Upper Right shows a Normal X-ray, and Bottom Left shows a Viral Pneumonia X-ray

\section{B. Building and training the model}

Google Colab is a free-to-use online terminal for python programming. It is widely used by students, data scientists and AI researchers on creating machine learning programs. Google Colab offers wide range of pre-installed libraries and allows user to save their projects on Cloud. They also provide free GPU and TPU to use, with a certain limitation to free accounts.

The Kaggle dataset is then import to the Google Colab interface, unzipped, and placed into directories. The images then are separated between dataset for images, x_dataset, and dataset for labels, y_dataset. Since the initial image size from the dataset is 299 x 299 pixels, the images are to be resized to $70 \times 70$ pixels. This is due to the reason where GPU does not accommodate large images along with large batch sizes, limiting the chances for a higher accuracy. The model uses train test split method, splitting $80 \%$ training data 
and $20 \%$ testing data. For this project, the model is attempted to be built by using Convolutional Neural Network (CNN).

CNN works by bedding pixels of a 2-Dimensional object into neurons in the input layer, multiplying the pixels to weights and their sum is sent as input to the neurons in the hidden layer. For this project, the model uses a simple neural network that is developed from the scratch with 3 convolutional networks, and 2 dropout layers in a sequential method. Sequential method here means that the layers of the model is arranged in a sequence.

After building the model, image augmentation is used to refine the model's accuracy. ImageDataGenerator is used to augment images while not affecting the original data. It creates new data so that the model can be trained with greater dataset, providing a better validation accuracy. ImageDataGenerator will be implied to both training and testing data, passing the data to training and testing directories.

To ensure a high accuracy for our model, epoch callbacks are necessary. Epoch is the number of successful passes after each training. Callback is a Keras function that includes stopping the model training after a certain accuracy or loss value is obtained and saving checkpoints of every epochs in the model. This feature eases the desire to gain the highest accuracy during training, saves time, as well as avoiding overfitting. Overfitting happens when training accuracy value is higher than testing accuracy, and may cause the model to predict wrongly, despite the high accuracy. In this case, the model is unfamiliar with new "testing" images, as it is too used with the trained images. Hence, a callback is used in this project. After the training process, final model will be saved, and testing loss and testing accuracy is evaluated.

\section{Visual Studio Code and Flask}

To produce a web server that can run uploading image and producing an output from the trained model that has been saved, Visual Studio Code is used to program Python, HTML and CSS environments for the web server. The web framework used is Flask, as Flask is written in Python, hence easier to configure along with the model.
Firstly, a python file is created. The model will be uploaded in the app,py file, and predict the uploaded image. As for the website's user interface, it is designed with a simple user interface that requires user to upload an X-ray image, and the displays the predicted outcome on the front of the web page itself. The code combines both HTML and CSS in one html file. By using Flask, the web framework is uploaded to localhost by running Flask in the terminal. Flask will then upload a link that will redirect to the local web server.

\section{RESULTS AND ANALYSIS}

\section{A. Model building: Image Augmentation}

As we all know, there are various methods on refining and the optimization of the model. One of the ways is to increase the image dataset with more augmented images. However, in some circumstances, image augmentation might not be the answer to a perfect fitted model. For instance, in this study, the detection of COVID-19 in X-ray images is detected by the amount of grey consolidation in the image [1]. If the augmented image is augmented to a Normal lung X-ray in a certain scale of zoom, the output will produce what seem to be a consolidated image.

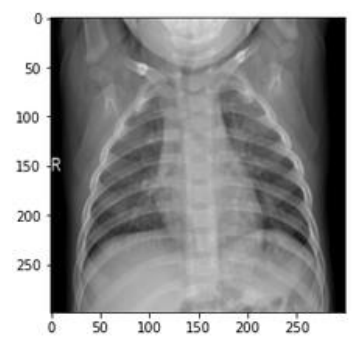

Figure 4. Normal X-ray which has not been augmented.

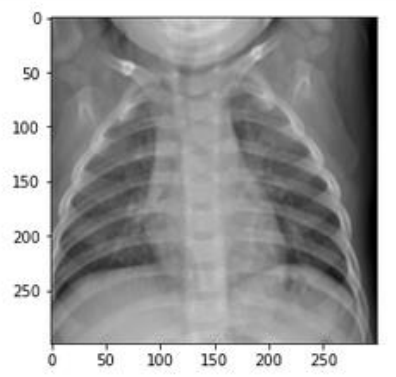

Figure 5. Augmented image of a Normal X-ray in the scale of 0.2 which appears to have blurrier (consolidation) amount, which could affect the detection. 
Hence, this issue increases loss values. Therefore, to achieve great results with expanding the data via image augmentation, specific choices of image augmentation is used to match with the detection method of COVID19 in X-ray images. Changes are made between the amount of zoom range, and brightness range. The reasons to why brightness range is chosen for image augmentation rather than zoom is because brightness could stretch the grayscale images of X-ray to many different scales of brightness without creating confusing training or testing dataset for the model to detect consolidation, unlike zooming.

The result bearing $87 \%$ validation accuracy for model's accuracy and loss is as shown below.

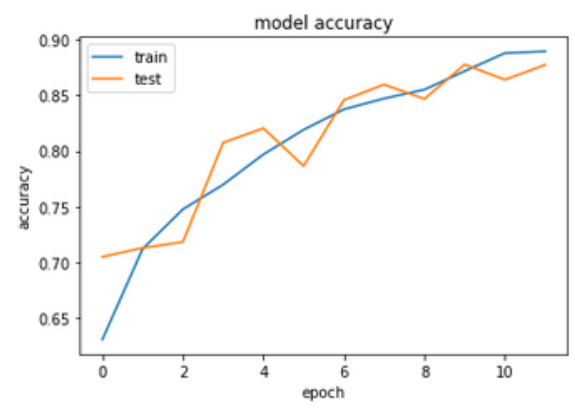

Figure 6. Testing and training accuracies for the chosen model.

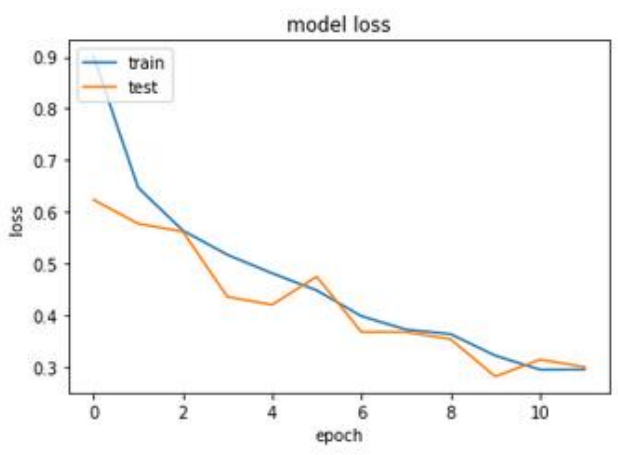

Figure 7: Testing and training loss for the chosen model.

\section{B. Batch sizes and image sizes}

Both batch size and image size are important to get an optimized accuracy value, and a perfectly fitted model. The average image size in the dataset is of 299x299 pixels. However, it seems that to the free GPU provided in Google Colab, it could not withstand huge images to be trained and tested, therefore sessions will crash. Therefore, to proceed with this study, smaller values of image size will be used, which is $70 \times 70$ pixels. Batch sizes are also crucial for the purposes of obtaining greater accuracy, as batch sizes is a hyperparameter that refers to amount of training in one iteration. In this study, 3 batch sizes are used for comparison ranging from 64 , 128 and 256. Batch size of 128 and the image size of $70 \times 70$ pixels, are chosen together with the configured image augmentation as they bear satisfying result of $85 \%$ validation accuracy.

As epochs are used with callbacks in this study, no adjustments with epochs are necessary to obtain the desirable value, as the epochs will stop once a desired value of loss and accuracy is achieved, to prevent overfitting issues.

\section{Applying to web server}

After applying the model to Visual Studio Code and running it in Flask, the web server requires user to upload image, and the model will provide a predicted result as shown below.

\section{COVID19 Recognition Server}

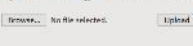

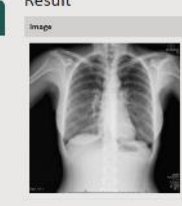

Figure 8: Predicted result from an uploaded image of a COVID-19X-ray.

\section{V) CONCLUSION AND RECOMMENDATION}

In this study, the presented idea of using machine learning to classify COVID-19 patients through X-ray images proves to be effective and beneficial for the future of humanity in facing the ever-evolving virus and pandemic. As results are analyzed, certain methods have been identified to specifically detect COVID-19 such as the limitations to image augmentation. This concludes that certain methods that are needed for model optimization are not necessary for X-ray image detection. As image sizes could not be experimented with using this dataset, at least the comparison between 
batch sizes is doable. The comparison made in this study is to obtain a simple CNN model with a satisfying value that could be implemented to a web server.

The local web server implemented also serves a great length to this study, as the study emphasizes the need of portability, operating procedures during the pandemic and ease of detection for radiologists. The simple user interface design serves straight-forwardness and ease of access to the users.

The study only limits to COVID-19 and Normal Xray images, and future works can be done by expanding on detecting viral pneumonia. Moreover, the study can be improvised by working the web server to an online service, or a private web server for organizations.

\section{REFERENCES}

[1]

J. Cleverley, J. Piper and M. Jones M, "The role of chest radiography in confirming covid-19 pneumonia," 16 July 2020. [Online]. Available:

https://www.bmj.com/content/370/bmj.m2426.

[2] H. Frank Wong, H. Sonia Lam, A. Ho-Tung Fong, S. Ting Leung, T. Wing-Yan Chin, C. Yen Lo, M. Mei-Sze Lui, J. Yin Lee, K. Wan-Hang Chu, T. Wai-Hin Chung, E. Phin Lee, E. Fai Wan, I. Ngai Hung, T. Wing Lam, M. Kuo and M. Ng, "Frequency and Distribution of Chest Radiographic Findings in Patients Positive for COVID-19," 27 March 2020. [Online]. Available: https://pubs.rsna.org/doi/full/10.1148/radiol.2020201160.

[3] S. Zhou, Y. Wang, T. Zhu and L. Xia, "CT Features of Coronavirus Disease 2019 (COVID-19) Pneumonia in 62 Patients in Wuhan, China," June 2020. [Online]. Available: https://www.ajronline.org/doi/full/10.2214/AJR.20.22975.

[4] K. Hyungjin, "Outbreak of novel coronavirus (COVID-19): What is the role of radiologists?," 30 June 2020. [Online]. Available:

https://www.ncbi.nlm.nih.gov/pmc/articles/PMC7220577/\#CR2.

[5] W. Gang and L. Xiaoming, "Mobile X-rays are highly valuable for critically ill COVID patients," 13 May 2020. [Online].
Available:

https://www.ncbi.nlm.nih.gov/pmc/articles/PMC7220577/\#CR2.

[6] Z. Chuansheng, D. Xianbo, F. Qiang, Z. Qiang, F. Jiapei, M Hui, L. Wenyu and W. Xinggang, "Deep Learning-based Detection for COVID-19 from Chest CTusing Weak Label," 2020. [Online]. Available: https://www.medrxiv.org/content/medrxiv/early/2020/03/26/2020 03.12.20027185.full.pdf.

[7] d. F. B. Valter Augusto, G. Juliana Carneiro, d. S. Maíra Araújo, d. A. A. Jeniffer Emidio, d. S. Rodrigo Gomes, d. S. Ricardo Emmanuel and d. S. Wellington Pinheiro, "Heg.IA: An intelligent system to support diagnosis of Covid-19 based on blood tests," 2020. [Online]. Available: https://www.medrxiv.org/content/10.1101/2020.05.14.20102533 v1.

[8] W. Jiangpeng, Z. Pengyi, Z. Liting, M. Wenbo, L. Junfeng, T. Chongxiang, L. Yonghong, C. Jing, Y. Zengwei, Z. Jinhong, Z. Meie, H. Huirong, X. Xiaodong and L. Shuyan, "Rapid and accurate identification of covid-19 infection through machine learning based on clinical available blood test results," 2020. [Online]. Available: https://www.medrxiv.org/content/10.1101/2020.04.02.20051136 v1.

[9] T. Ozturk, M. Talo, E. Yildrim, U. Balogu, O. Yildrim and U. Acharya, "Automated detection of COVID-19 cases using deep neural networks with X-ray images," 28 April 2020. [Online]. Available:

https://www.ncbi.nlm.nih.gov/pmc/articles/PMC7187882/.

[10] C. Joseph Paul, M. Paul, D. Lan, R. Karsten, D. Tim Q and G. Marzyeh, "GitHub COVID-19 image data collection," [Online]. Available: https:/github.com/ieee8023/covid-chestxray-dataset.

[11] T. Enzo, B. Carlo Alberto, B. Claudio, C. Marco and G. Marco, "Unveiling COVID-19 from Chest X-ray with deeplearning: a hurdles race with small data," 11 April 2020. [Online]. Available: https://arxiv.org/pdf/2004.05405.pdf.

[12] S. Prabira Kumar, B. Santi Kumari, R. Pradyumna Kumar and B. Preesat, "Detection of coronavirus Disease (COVID-19) based on Deep Featuresand Support Vector Machine," [Online]. Available:

https://www.preprints.org/manuscript/202003.0300/v2.

[13] T. Rahman, "COVID-19 Radiography Database," [Online]. Available: https://www.kaggle.com/tawsifurrahman/covid19radiography-database. 